\title{
Self-improving collodion baby
}

INSERM

\section{Source}

INSERM. (1999). Orphanet: an online rare disease and orphan drug data base. Selfimproving collodion baby. ORPHA:281122

Self-healing collodion baby (SHCB) is a minor variant of autosomal recessive congenital ichthyosis (ARCl; see this term) characterized by the presence of a collodion membrane at birth that heals within the first weeks of life. 